\title{
Pour une approche genrée du mensonge
}

Für ein genderorientiertes Lügenmodell

For a gender approach to lies

\section{Susanne Böhmisch}

\section{OpenEdition}

\section{Journals}

Édition électronique

URL : http://journals.openedition.org/ceg/1507

DOI : $10.4000 /$ ceg. 1507

ISSN : 2605-8359

\section{Éditeur}

Presses Universitaires de Provence

Édition imprimée

Date de publication : 15 juin 2015

Pagination : 149-163

ISBN : 978-2-85399-993-9

ISSN : 0751-4239

\section{Référence électronique}

Susanne Böhmisch, «Pour une approche genrée du mensonge », Cahiers d'Études Germaniques [En ligne], 68 | 2015, mis en ligne le 17 décembre 2017, consulté le 06 décembre 2020. URL : http:// journals.openedition.org/ceg/1507 ; DOI : https://doi.org/10.4000/ceg.1507 


\section{Pour une approche genrée du mensonge} Susanne BÖHMISCH

Aix Marseille Université

Comment la sémantique du mensonge affecte-t-elle les discours normatifs sur le genre? En quoi l'histoire du mensonge est-elle liée à l'histoire de la différence sexuelle et à son interprétation ${ }^{1}$ ? En quoi l'histoire de la différence sexuelle peut-elle nous éclairer sur les différentes interprétations du mensonge, sur le degré de tolérance à son égard, sur l'échelle des sanctions? Si le menteur est un sujet sexué, le mensonge est-il genré? Voilà des questions soulevées par la thématique mensonge et genre. Dans notre contribution, nous tenterons de poser quelques jalons en nous concentrant principalement sur les figures féminines du mensonge et de la ruse. Il s'agira dans un premier temps de rappeler le démasquage des mensonges sur le genre, ce qui permettra de délimiter notre thématique et de problématiser la notion d'intentionnalité - élément constitutif dans la définition classique du mensonge -, par rapport au genre. Dans un second temps, nous montrerons ce qui, chez les deux philosophes féministes ayant le plus marqué le $\mathrm{xx}^{\mathrm{e}}$ siècle, à savoir Simone de Beauvoir et Judith Butler, peut appuyer notre argumentaire. Enfin, l'exemple du déplacement de la ruse d'Hérodiade à la danse de Salomé permettra de saisir comment la construction du genre se modifie en fonction de l'interprétation de la ruse féminine.

\section{Démasquer les mensonges sur le genre}

Qu'il y ait du mensonge dans les discours sur le genre, ou du moins dans les représentations normatives du genre, est une critique largement répandue depuis un demi-siècle de réflexion féministe et d'études dans le champ des gender studies. Une visée majeure de cette critique est de dénoncer toutes les tentatives de naturalisation du genre, de montrer en quoi les définitions du féminin et du masculin relèvent d'une construction socio-culturelle, et ne constituent point une vérité ontologique. Nombreuses sont les approches théoriques et les études dans un grand nombre de disciplines ayant dénoncé ce qui dans ces constructions serait mensonger. Dans son essai de 1970, Die endlose Unschuldigkeit (L'Innocence sans fin), Elfriede

\footnotetext{
1 Jacques DerRIDA avait évoqué leur lien brièvement dans Histoire du mensonge: « [I]1 y aurait plus d'une conférence à consacrer à ce qui lie l'histoire du mensonge à l'histoire de la différence sexuelle, de son érotique et de ses interprétations ». Paris, Galilée, 2012, p. 45.
} 
Jelinek utilisa l'expression forte d'une "viscosité du naturel qui couvre tout» (« natürlichkeitsschleim, der alles überzieht $\left.{ }^{2} »\right)$. Tout son projet de démythification inspiré par le mythe moderne au sens barthésien consiste à démasquer l'idéologie patriarcale, capitaliste, néo-libérale qui se niche dans tout ce qui se prétend naturel, y compris en matière de genre. Son œuvre peut être considérée aujourd'hui comme une des déconstructions les plus radicales de toutes les tentatives mensongères de naturalisation ainsi que de récupération du genre.

Mais se trouve-t-il quelqu'un qui mente consciemment, qui établisse une contrevérité sur le genre, en sachant qu'il ment? Où situer l'intentionnalité? Gotthold Ephraim Lessing par exemple, dans sa polémique contre la Rodogune de Pierre Corneille, soutient en 1767 qu'une femme comme Cléopâtre, qui n'hésite pas à assassiner son époux et ses fils, et dont la ruse infâme est motivée uniquement par le pouvoir, serait un «monstre dans son $\operatorname{sexe}^{3} »$. Lorsque, ce faisant, il impose au nouveau drame bourgeois son propre idéal de féminité qu'il situe du côté de la vertu, de la douceur, du naturel et de la sincérité, il ne pense pas mentir, au contraire, il pense affirmer la vérité sur la femme, et nul doute qu'il ne le croie vraiment ${ }^{4}$. Et pourtant, la norme qu'il établit ainsi sera, comme le montre Peter von Matt dans son étude sur l'intrigue, un geste fondateur du drame bourgeois et, par ailleurs, des représentations normatives de la femme depuis la fin $\mathrm{du} \mathrm{XVIII}^{\mathrm{e}}$ siècle, celles que les études féministes du $\mathrm{XX}^{\mathrm{e}}$ siècle prendront comme cible dans leur critique des mensonges sur le genre. S'il est parfois relativement aisé de mettre en évidence en quoi les représentations normatives ont produit beaucoup de fausses vérités sur le genre, il est souvent moins aisé de montrer qui ment en premier, de prouver son intentionnalité et de faire voir comment les mensonges se fabriquent.

De surcroît, le critère de l'intentionnalité s'effrite avec la modernité, notamment suite à la crise du langage et du sujet, et grâce à la psychanalyse. Nous savons que l'on peut mentir sans en avoir l'intention et que l'on peut dire la vérité du sujet tout en essayant de la dissimuler. Ainsi, il y a eu au $\mathrm{Xx}^{\mathrm{e}}$ siècle, dans l'histoire du mensonge, de nombreuses tentatives de s'affranchir du critère de l'intentionnalité, notamment dans les réflexions poétologiques entre les années 60 et 90 . Ingeborg Bachmann par exemple considère comme langage mensonger et condamnable le langage figé, représentant d'un ordre conservateur qu'elle nomme "langue de brigand» («Gaunersprache $\left.{ }^{5} »\right)$ en 1961. Doren Wohlleben commente cette définition moins classique du mensonge ainsi :

2 Elfriede JelineK, Die endlose Unschuldigkeit, Schwifting, Schwiftinger Galerie-Verlag, 1980 [1 ${ }^{\text {re }}$ éd. 1970$]$, p. 56.

3 « ein Ungeheuer ihres Geschlechts », G.E. Lessing, Hamburgische Dramaturgie, Stuttgart, Reclam, 1981, n ${ }^{\circ} 29$ à 32, ici n 30 (35 soirée), p. 159.

4 Voir l'ouvrage de Peter von Matt, Die Intrige. Theorie und Praxis der Hinterlist, München, Hanser Verlag, 2006, ainsi que l'article d'Ingrid HAAG dans le premier volume des Cahiers d'Études Germaniques consacré au mensonge: "Über die "Wahrheit" der weiblichen Natur und wie diese auf der Bühne des bürgerlichen Trauerspiels Lügen gestraft wird », " Quelques vérités à propos du mensonge? ", vol. I, Cahiers d'Études Germaniques, n 67, 2014, p. 123-136.

5 Ingeborg BaChMAnN, Das dreißigste Jahr, in Ingeborg Bachmann: Werke, éd. par Christine Koschel, Inge von Weidenbaum, Clemens Münster, œuvres complètes en quatre volumes, ici vol. II, München/ Zürich, Piper, 1978, p. 94-137, ici p. 112. 
Die Lüge wird mit einer alten, phrasenhaften Sprache, einer zur Veränderung nicht gewillten Ordnung assoziiert, vehement abgelehnt und gilt als moralisch verwerflich. Sie bildet $[\ldots]$ einen dichotomischen Gegenpol zu einer positiv konnotierten, utopischen Wahrheit, der sich Literatur approximativ zu nähern habe. [...] Nicht mehr die Behauptung falscher Tatsachen bedingt also den Lügenvorwurf, sondern der Gebrauch überkommener, verlogener Ausdrücke. Dies hat zur Folge, daß die Lüge nicht, wie die Definition von Augustin dies noch voraussetzte, intentional gefaßt werden kann. ${ }^{6}$

Dans cette « langue de brigand », dénoncée par Ingeborg Bachmann, figurent les normes du genre d'une idéologie patriarcale, ces normes que nous nous approprions et que nous rejouons lors de nos processus de constructions identitaires. Par conséquent, bien qu'il n'y ait pas toujours, et probablement même rarement intention de mentir, des normes sur le genre sont constamment produites qui finissent par instaurer des fausses vérités, des mensonges, une "langue de brigand sur le genre" pourrait-on dire, qu'un demi-siècle de gender studies a déjà largement analysée.

\section{La menteuse et le pouvoir (Simone de Beauvoir)}

En ce qui concerne un lien discursif plus étroit et structurel entre mensonge et genre, il est intéressant de considérer la figure de la menteuse. Que la femme soit menteuse, qu'elle partage avec le diable la duplicité et la ruse, qu'elle ne cesse de tromper l'homme, est un stéréotype bien connu. Il semble perdurer jusque dans la modernité de nos sociétés sécularisées. Voici ce qu'en dit S. de Beauvoir en 1949:

[La femme] n'a pas le sens de la vérité, ni de l'exactitude, elle manque de moralité, elle est bassement utilitaire, elle est menteuse, comédienne, intéressée... Il y a dans toutes ces affirmations une vérité. Seulement les conduites que l'on dénonce ne sont pas dictées à la femme par ses hormones ni préfigurées dans les cases de son cerveau: elles sont indiquées en creux par sa situation. ${ }^{7}$

Dire que la femme est menteuse, comédienne, n'est donc pas faux. Mais S. de Beauvoir se garde bien d'affirmer qu'il s'agirait là d'une vérité ontologique. Elle s'oppose nettement aux thèses - très fréquentes autour de 1900 - qui parlent d'une 《 nature mensongère ${ }^{8}$ » de la femme. La tendance au mensonge et à la dissimulation s'explique pour S. de Beauvoir en 1949 par la «situation » de la femme, à savoir le rôle subalterne qu'elle joue, confinée dans le champ de l'immanence. Selon elle, beaucoup des défauts qu' on reproche aux femmes « expriment simplement le fait que l'horizon leur est barré ${ }^{9} »$. Si l'homme la rêve idéale et infinie, s'il la mythifie sous la figure de l'éternel féminin, il lui assigne en même temps la médiocrité de l'espace domestique. Pourtant, lorsqu'elle ne correspond pas à son idéal, il lui reproche de ne

6 Doren Wohlleben, Schwindel der Wahrheit. Ethik und Ästhetik der Lüge in Poetik-Vorlesungen und Romanen der Gegenwart, Freiburg/ Berlin, Rombach, 2005, p. 211-213.

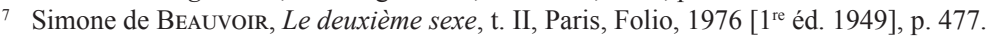

8 Cf. « die ontologische Verlogenheit des Weibes », Otto WeInINGER, Geschlecht und Charakter,

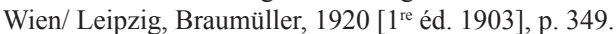

9 Beauvoir, Le deuxième sexe, t. II, p. 485. 
pas être féminine ou de le tromper ${ }^{10}$. Toute incohérence avec son idée de féminité à lui sera imputée à elle, nourrira le soupçon à son égard, elle qui de toute façon est toujours soupçonnable. Et la femme se découvre elle-même « comme mensonge ${ }^{11}$ », écrit S. de Beauvoir. Si l'homme érige comme idéal la femme vertueuse, la « Femme sans ruse ${ }^{12}$ ", il cherche aussi son contraire: "L'homme lui assène pompeusement son code de vertu et d'honneur; mais en douce il l'invite à y désobéir ${ }^{13}$. » La prostitution serait un exemple parmi d'autres de cette hypocrisie masculine.

Selon S. de Beauvoir, la femme n'est pas dupe et a bien compris cette mystification dont elle fait l'objet, cette assignation à la place de l'Autre, la fonction d'écran du fantasme masculin. Cette assignation impose à la femme soit de jouer l'Autre ("L'homme même réclame qu'elle lui joue une comédie: il veut qu'elle soit $l^{\prime}$ Autre $\left.{ }^{14} \gg\right)$, soit de ruser pour échapper à cette emprise, à cette dépendance ( « c'est seulement par le mensonge et l'adultère qu'elle peut prouver qu'elle n'est la chose de personne et qu'elle dément les prétentions du mâle $\left.{ }^{15} »\right)$. Dans les deux cas, les comportements féminins confirment à nouveau les soupçons à son égard, comédienne et menteuse, et c'est donc la femme elle-même qui se fait complice de ces soupçons.

Si S. de Beauvoir décrit de nombreuses scènes empiriques de son époque où l'homme demande à la femme de feindre passivité, jouissance ou indépendance, ou d'autres où la femme ment pour retenir l'homme ou se moquer de lui, c'est pour réaffirmer que tous ces comportements ne constituent guère une vérité ontologique. Pour elle, citant d'abord Charles Fourier: "Il y a fausseté partout où il y a régime coercitif ${ }^{16} »$, puis se référant aux racistes américains et aux colons français pour qui le Noir est nécessairement paresseux et menteur, car « il prouve par là son indignité; il met le bon droit du côté des oppresseurs ${ }^{17} »$, ce type de mensonge genré doit plutôt se comprendre comme manifestation d'un rapport de pouvoir.

Le mensonge n'est pas seulement un stratagème de vanité, comme nous le dit Friedrich Nietzsche. Il sert aussi un pouvoir ou il est tentative de se soustraire à un pouvoir. Si le reproche de mensonge adressé à l'autre est employé par l'oppresseur pour asseoir sa souveraineté, le mensonge est aussi utilisé par les opprimés pour échapper au contrôle et à la domination. Il est un moyen d'affirmation de soi pour ceux et celles dont la position de sujet est menacée, des femmes et des hommes marginalisé-e-s pour des raisons culturelles, idéologiques, politiques ou d'orientation

10 «Ainsi, à l'existence dispersée, contingente et multiple des femmes, la pensée mythique oppose l'Éternel Féminin unique et figé; si la définition qu'on en donne est contredite par les conduites des femmes de chair et d'os, ce sont celles-ci qui ont tort: on déclare que la Féminité est une entité, mais que les femmes ne sont pas féminines. Les démentis de l'expérience ne peuvent rien contre le mythe. » Le deuxième sexe, t. I, p. 395.

11 Ibid., p. 306.

12 Simone de Beauvorr cite ici des litanies du Moyen Âge, adressées à la Vierge Marie (« Tu es la Femme sans ruse dont l'amour jamais ne change... »). Ibid., p. 297.

13 Beauvoir, Le deuxième sexe, t. II, p. 497.

14 Ibid., p. 499.

15 Beauvoir, Le deuxième sexe, t. I, p. 309.

${ }^{16}$ Beauvorr, Le deuxième sexe, t. II, p. 500.

${ }^{17}$ Ibid., p. 501. 
sexuelle. Nous pouvons à ce propos nous reporter aux thèses de Vladimir Jankélévitch, pour qui un ressort important du mensonge est le fait d' « d'agrandir l'espace vital de son ego ${ }^{18} \gg$. Comme posture face au pouvoir, mensonge et ruse se rapprochent pour Jankélévitch. Ce sont les armes des faibles: « le menteur ruse avec la difficulté [...]; il simule et dissimule afin d'obtenir, en trichant, de petites rectifications de frontière ${ }^{19} »$. Ruser, mentir reviendrait donc à essayer de gagner en espace vital, et simultanément cela participe activement à la construction et la confirmation des stéréotypes, par exemple sur la menteuse.

S. de Beauvoir ne dit pas autre chose: un agir, ici le fait de mentir, résulte d'une situation sociale, existentielle complexe; il finit par produire un réel normatif, et la femme devient menteuse. C'est le pourquoi et le comment de ces situations, la fabrique du genre dans l'histoire du mensonge, qui est intéressant à analyser, et tout particulièrement le lien étroit entre la sémantique du mensonge et l'exercice du pouvoir. Si mensonge et manipulation se trouvent certes du côté des dominants, le fait de faire peser sur quelqu'un le soupçon de mensonge (ce dernier étant considéré comme moralement condamnable), est aussi un acte de domination. Confondre "situation" et "nature" de la femme, affirmer que toutes les femmes seraient "par nature" des menteuses, c'est aussi discréditer leur parole, les priver de légitimité et participe donc activement à la hiérarchisation de la différence sexuelle.

\section{La performativité de la menteuse (Judith Butler)}

Plus près de nous, les réflexions butleriennes sur le rapport entre norme et performativité, norme et pouvoir, ainsi que la notion de resignification peuvent appuyer notre argumentaire. Rappelons d'abord ce que J. Butler dit à propos des normes et de la performativité. Dans son ouvrage Défaire le genre, elle distingue la norme de la loi, notamment par sa dimension diffuse:

Les normes peuvent être explicites ou non et, lorsqu'elles opèrent en tant que principe normalisateur dans les pratiques sociales, elles restent habituellement implicites, difficiles à décrypter, et il n'est pas simple de discerner clairement les effets qu'elles produisent ${ }^{20}$.

Le genre est pour J. Butler une norme et donc « toujours et uniquement faiblement incorporé par n'importe quel acteur social ${ }^{21} »$. Ce sont les normes qui font que nos actions sont reconnaissables, que le sujet devient lisible au niveau social ${ }^{22}$. Si vous vous détournez des normes, vous risquez de devenir un sujet illisible ou rejeté ${ }^{23}$.

18 Vladimir JankÉLÉVITCH, Traité des Vertus II, t. 1, « Les vertus et l'amour », Paris, Flammarion, 1986, p. 196.

${ }_{19}$ Ibid., p. 198.

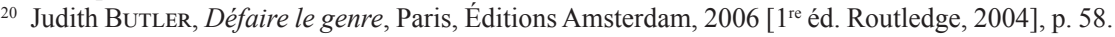

${ }^{21}$ Ibid., p. 59.

22 Ibid.

23 « [L] es personnes sont régulées par le genre et $[\ldots]$ ce type de régulation opère comme une condition de l'intelligibilité culturelle de chacun. Se détourner de la norme de genre revient à produire l'exemple aberrant que les pouvoirs régulateurs (médical, psychiatrique et légal, pour en nommer quelques-uns) peuvent rapidement exploiter pour justifier leur propre zèle régulateur continu. » Ibid., 
Mais le genre est aussi un acte performatif où le sujet s'approprie, réitère, rejoue les normes discursives et culturelles qui circulent à un moment donné dans la société. Il a donc sa part de responsabilité ainsi qu'une marge de subversion possible. C'est la thèse qu'elle soutient dans Gender Trouble où elle dénonce le caractère contraignant et comique de la norme hétérosexuelle. Ce qu'elle nomme, dans sa relecture de Jacques Lacan, la « comédie hétérosexuelle ${ }^{24}$ », ne serait qu'une fiction, une simulation, une performance. L'exemple de la drag-queen devient l'emblème même de ces mécanismes de simulation, de cette performance du genre qui, selon elle, opère à tous les niveaux. La drag-queen rend visibles les apparences trompeuses liées au genre et une discontinuité entre sexe, genre et sexualité. Voici ce que J. Butler en dit quelques années plus tard dans une interview:

\begin{abstract}
Dans ce livre, j'ai utilisé l'exemple de la drag-queen pour établir que la performance du genre qu'une drag-queen propose n'est pas moins réelle ou moins vraie que celle qu'effectue [perform] un homme ou une femme quelconque, qu'il s'agit d'une sorte d'allégorie de la performance ordinaire du genre, et que nous effectuons pour ainsi dire tous, tout le temps, le genre. Le spectacle drag constitue un moment où la performance est rendue explicite. Ce n'est pas un écart aberrant par rapport à la norme; il expose la façon dont la norme fonctionne effectivement, la façon dont elle est instituée au travers du corps, de notre stylistique, de nos expressions corporelles ${ }^{25}$.
\end{abstract}

Une thèse fondamentale de J. Butler est en effet de dire: Je ne peux être sans faire. Mais mon faire dépend de ce qu'on me fait - des « manières dont je suis faite par les normes ${ }^{26}{ }$ - et de ma capacité à faire quelque chose avec ce qu'on me fait, ce qu'elle nomme agency, puissance d'agir.

On pourrait donc émettre l'hypothèse suivante: la sémantique du mensonge, faisant partie des normes discursives et culturelles, impacte la construction $\mathrm{du}$ genre et la performativité du sujet. Les analyses de S. de Beauvoir sur la menteuse montrent bien comment une telle réglementation du genre se met en place. S'il y a une différenciation sexuée entre menteur et menteuse, une différence quant aux seuils de tolérance ou sanctions à l'égard du menteur ou de la menteuse, cette interprétation du mensonge aura un impact sur le faire du sujet.

Voici quelques exemples propres à esquisser l'historicité du lien discursif entre mensonge et genre entre le $\mathrm{XVIII}^{\mathrm{e}}$ et le $\mathrm{XX}^{\mathrm{e}}$ siècle. L'idéal de sincérité qui, à partir de la fin du XVIII ${ }^{\mathrm{e}}$ siècle dans les pays germanophones, affecte la femme, notamment dans la communication intime (elle doit être transparente et lisible), constitue un important moyen de contrôle, une véritable stratégie répressive. S'appuyant sur les réflexions de Michel Foucault à propos de l'aveu, J. Butler écrit: « [C]'est par la confession que les discours d'autorité peuvent nous contrôler. Nous disons ce que nous avons pensé ou fait, et cette information devient le matériau grâce auquel nous

\footnotetext{
p. 70.

24 « Komödie der Heterosexualität », Judith Butler, Das Unbehagen der Geschlechter, Frankfurt a.

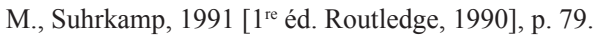

25 Judith ButLer, "Changer le sujet: la resignification radicale », in Humain, Inhumain. Le travail critique des normes. Entretiens, Paris, Éditions Amsterdam, 2005, p. 93-145, ici p. 124.

${ }^{26}$ ButLer, Défaire le genre, p. 15.
} 
sommes interprétés ${ }^{27} \gg$. Interdire le mensonge reflète une forte volonté de maîtrise. Dans la mesure où il porte sur des domaines différents puisque le clivage entre privé et public, entre communication intime et discours politique ou philosophique est en train de s'institutionnaliser, il constitue également une réglementation du genre. Face à cet impératif de dire la vérité (de ses émotions et pensées), assez tyrannique en soi, le sujet féminin, s'il veut sauver quelque chose de son jardin secret, doit mettre en œuvre des stratégies particulièrement rusées afin de continuer à paraître le plus sincère possible. L'impératif de tout dire le pousserait donc à s'exercer dans l'art de la dissimulation et de la ruse. Et lorsqu'il s'agit d'accéder au statut d'auteure, d'écrivaine, et de dire ce qui ne convient pas à une parole de femme, le recours au pseudonyme masculin s'impose pour de nombreuses femmes à cette période et tout au long du XIX ${ }^{\mathrm{e}}$ siècle $^{28}$.

Si l'on analyse les théories misogynes sur la nature mensongère de la femme, fréquentes autour de 1900, notamment dans la modernité viennoise où aussi bien la question sexuelle que le mensonge préoccupent les esprits, on constate une tendance à faire sortir le mensonge du champ moral et à le réhabiliter, après F. Nietzsche, mais aussi à reléguer la femme dans la variante pathologique du mensonge, l'hystérisation de la femme étant à son comble ${ }^{29}$. Ce qui peut toutefois étonner, dans ces mêmes écrits misogynes, c'est qu'ils manifestent fréquemment une sorte de tolérance à l'égard des mensonges de femmes, à la seule condition que ceux-ci servent à ce que les femmes jouent bien leur rôle clairement défini dans la hiérarchie des sexes. Chez Paul Möbius par exemple, nous lisons :

\begin{abstract}
Die Aufgabe ist, begehrenswerth zu erscheinen, deshalb muss das eigene Begehren verschwiegen werden, und muss alles geschickt verdeckt werden, was der Schätzung der Anderen abträglich sein könnte. [...] nichts ist thörichter als dem Weibe das Lügen verbieten zu wollen. Verstellung, d.h. Lügen, ist die natürliche und unentbehrlichste Waffe des Weibes, auf die sie gar nicht verzichten kann. Freilich soll die Waffe nur zur Vertheidigung dienen, indessen ist es begreiflich, dass es nicht dabei bleibt, dass ein Verfahren, das einen wichtigen Theil der Lebensführung bildet, auch ohne Noth angewendet wird. An sich ist die weibliche Lüge nur in geschlechtlichen Beziehungen gerechtfertigt, die Billigkeit aber fordert, dass sie überhaupt milder beurtheilt werde als die männliche Lüge. ${ }^{30}$
\end{abstract}

L'argumentation de base semble remonter à Jean-Jacques Rousseau: La femme a une libido puissante, mais elle doit la masquer afin de ne pas effrayer l'homme et de lui plaire ${ }^{31}$. Elle pratique donc l'art de la dissimulation que P. Möbius, tout autant

${ }^{27}$ Ibid., p. 188.

${ }_{28}$ Dans une étude sur les pseudonymes avec changement de genre dans l'espace germanophone entre 1700 et 1900, Susanne KoRD a recensé 482 cas d'écrivaines avec un pseudonyme masculin contre 12 cas d'écrivains avec un pseudonyme féminin, ces derniers étant motivés par l'intention soit d'adresser des leçons de morale aux femmes, soit de s'inscrire dans un genre littéraire connoté féminin comme le journal intime ou le roman épistolaire. Cf. Susanne Kord, Sich einen Namen machen. Anonymität und weibliche Autorschaft 1700-1900, Stuttgart/ Weimar, Metzler, 1996, p. 200.

${ }^{29}$ Voir Susanne Böнmisch, "Eines ist mir klar: Daß die Weiber auch in der Hypnose lügen". Mensonge et genre chez Schnitzler », Cahiers d'Études Germaniques, nº 67, p. 169-184.

${ }^{30}$ Paul MöвIUs, Über den physiologischen Schwachsinn des Weibes, Hamburg, Mattheus \& Seitz, 1990 [1 $1^{\text {re }}$ éd. 1908], p. 40.

31 Sur la ruse féminine chez Jean-Jacques Rousseau, voir la contribution de Friederike Kuster dans 
qu'Otto Weininger ou même F. Nietzsche, circonscrivent aux rapports sociaux de sexe et considèrent comme typiquement féminin. Ce qui retient notre attention dans cette citation, c'est que les mensonges des femmes devraient être moins sanctionnés que les mensonges des hommes, puisqu'ils servent un ordre que Möbius considère comme naturel, à savoir la femme comme être sexué et inférieur qui veut et doit plaire à l'homme. Mais gare à la femme qui ose, par le mensonge, troubler ce cadre et cette hiérarchie ! Un tel discours, s'il est relayé massivement et popularisé, comme ce fut le cas pour les thèses d'O. Weininger, a des effets normatifs et un impact sur le faire de la femme. Il suggère implicitement que peu importe si elle ment ou dit la vérité, de toute façon, sa parole ne vaut rien, elle n'est pas capable de vérité. Elle est invitée à jouer, dissimuler, mentir, au service de son sexe. Sa performativité comme menteuse serait donc directement liée au fonctionnement de ces normes. Face à une telle déconsidération de sa parole, il n'est guère surprenant de voir la femme répliquer par un recours généreux au mensonge et à la ruse. D'ailleurs, pourquoi celle que certains stigmatisent comme immorale se sentirait-elle freinée par des considérations morales au sujet du mensonge?

Enfermer quelqu'un dans l'image du menteur ou de la menteuse est un acte par lequel s'affirme une autorité, une souveraineté qui cherche à imposer sa loi. C'est un acte qui peut servir de régulation du genre, comme ce fut le cas dans le discours sur l'hystérique, figure emblématique de la grande menteuse au $\mathrm{xx}^{\mathrm{e}}$ siècle, et donc du mensonge féminin dans sa variante pathologique. Georges Didi-Huberman, dans son ouvrage sur l'iconographie photographique de la Salpêtrière, avait dénoncé l'«hypocrisie» de Charcot, qui, sous prétexte de rendre visible la pathologie hystérique et de dire la vérité à son propos, l'aurait mise en scène, interprétée, inventée ${ }^{32}$. Charcot se serait doté pour cela d'une arme moderne: la photographie, considérée à l'époque comme garante de vérité et d'objectivité. Les images étaient censées constituer un tableau clinique, une mémoire, elles devaient donner à voir les différents faciès et gestes de la pathologie hystérique. G. Didi-Huberman montre au contraire que cette iconographie photographique relève d'une dramaturgie savamment orchestrée, qu'elle sert surtout à donner la preuve de la validité des thèses de Charcot sur l'hystérie ${ }^{33}$. Il repère même une "complicité médicopolicière » dans ces tentatives de répertorier, classifier et lire l'identité de quelqu'un dans une image ${ }^{34}$. Charcot a donc établi un type général d'hystérie, il a créé non seulement un tableau "clinique" mais aussi "classique", qui fit autorité par la suite et

ce même volume: " "Durch die List ist der Willen zum Weiblichen geworden." Bemerkungen zu einer Stelle aus Hegels Jenaer Systementwürfen ».

32 Georges Didi-Huberman, Invention de l'hystérie. Charcot et l'iconographie photographique de la Salpêtrière, Paris, Éditions Macula, 2012 [1 1 éd. 1982], p. 24.

${ }^{33}$ Georges Didi-Huberman dénonce dans son ouvrage ces tromperies où les médecins provoquaient de façon expérimentale des crises hystériques afin de photographier, observer et classifier les expressions des patientes. Une excitation des nerfs par exemple, ou l'administration de médicaments hallucinogènes, amenait des contractures et déclenchait une crise. Du coup expérimental on passe à l'attitude, de l'attitude au tableau de l'hystérie. En plus, la mémoire du coup expérimental est effacée puisque le médecin n'apparaît jamais sur les photos. Ce qu'on nous donne à voir semble d'autant plus 'naturel', 'ontologique' et typiquement féminin. Ibid., p. 275-277.

${ }^{34}$ Ibid., p. 79. 
auquel d'autres psychiatres se sont référés. Augustine fut son "chef-d'œuvre”, le cas hystérique parfait car absolument "régulier". Il la considéra « comme une honnête hystéro-épileptique, ni "farceuse", ni "styleuse": régulière, donc ${ }^{35} »$. Elle a gagné l'attribut d' " honnête » puisque ses crises correspondaient exactement au protocole de ses quatre phases de l'attaque hystérique ${ }^{36}$.

Comme beaucoup d'autres avant lui, Charcot a voulu donner une loi à cette pathologie sans loi. G. Didi-Huberman précise que c'est le symptôme hystérique lui-même qui est désormais soupçonné de mensonge, puisqu'il ne repose sur rien, ce qui aurait été insupportable à la médecine :

Que le fou soit celui qui ait perdu le sens de sa vérité, que les lois du monde lui échappent, et même les lois de sa propre essence, cela reste concevable. Mais qu'une femme fasse mentir son propre corps! Comment la médecine peut-elle continuer à s'exercer honnêtement, si les corps eux-mêmes se mettent à mentir ${ }^{37}$ ?

Dans notre perspective d'analyse, il ne faut pas sous-estimer la grande popularité dont a joui Charcot, ainsi que la vulgarisation de l'hystérie. Ces idées sur les femmes qui savent si bien mentir, qui savent même faire mentir leur corps au point de provoquer des symptômes ne reposant sur rien, ont pu ainsi atteindre un grand public et participer à la construction des normes du genre, ici à la pathologisation de la menteuse. En 1890, Charcot entrait au petit panthéon d'une Bibliothèque des merveilles qui connaissait une diffusion importante ${ }^{38}$. Même les magiciens dans les cirques ambulants de l'époque se référaient à lui pendant leurs spectacles d'hypnose ce qui évidemment ne l'enchantait guère. G. Didi-Huberman affirme que l' " hystérisation [...] du corps de la femme persiste et se refabrique même au $\mathrm{XIX}^{\mathrm{e}}$ siècle $^{39}$ ». Les soupçons de mensonge à l'égard de la femme se renforcent et aboutissent à une forte défiance dans la période hautement ambivalente autour de 1900 où la poussée émancipatrice des femmes va de pair avec un antiféminisme puissant. Si la femme fatale a été à ce moment-là l'une des représentations majeures, c'est aussi en raison de cette hystérisation du corps de la femme, en raison des soupçons à l'égard de ce corps qui peut mentir. Ces soupçons ont comme renforcé l'énigme-femme et renforcé le reproche du mensonge, adressé facilement à l'autre quand celui-ci vous échappe, comme l'a montré Thomas Keller dans le contexte de l'interculturalité ${ }^{40}$.

Le genre est un acte performatif. Le sujet peut réitérer les normes discursives ou bien les déjouer. Et il peut produire ce que J. Butler appelle une resignification: changer le sens d'un mot, notamment d'une insulte. J. Butler développe ce concept dans son

${ }^{35}$ Ibid., p. 164.

36 Ibid., p. 157.

37 Ibid., p. 105.

${ }^{38}$ Gustave Hahn, "Charcot et son influence sur l'opinion publique », Revue des questions scientifiques, $2^{\mathrm{e}}$ série, VI, 1894, p. 230-261 et 353-379. Cité par DiDI-HubERman, Invention de l'hystérie, p. 310 .

39 Didi-Huberman, Invention de l'hystérie, p. 113.

40 Thomas Keller, « Über Wahrheit und Lüge jenseits des deutsch-französischen Sinns », Cahiers d'Études Germaniques, nº 67, p. 271-288. 
ouvrage sur les mots qui blessent, Le pouvoir des mots. Politique du performatif ${ }^{41}$. Elle croit au pouvoir des mots qui désamorcent la haine, par un détournement où le sujet regagne en puissance d'agir. Un exemple désormais emblématique d'une telle réévaluation serait le terme queer qui signifiait d'abord de façon péjorative l'homosexuel et dont s'est emparé la communauté afin de lui donner un sens positif ${ }^{42}$. Une même tentative de réhabiliter un terme fortement péjoratif et genré, à savoir « sorcières », a été réalisée par des féministes dans les années $70^{43}$. L'idée d'une resignification pourrait être transférée sur le mensonge: lorsque le reproche de mensonge est utilisé dans un but de dévaloriser, voire d'opprimer l'autre, lorsqu'une morale cherche à s'imposer en reprochant à l'autre de proférer des mensonges, ceux et celles qui se retrouvent sur le banc des accusé-e-s peuvent s'emparer du terme et le retourner contre ceux qui le prononcent, mettant en échec l'opposition entre vérité et mensonge.

Il est évident que le rôle de la littérature et des arts est primordial dans cette « chaîne rituelle de resignifications » par laquelle s'accomplit, selon J. Butler, une " performativité discursive ${ }^{44}$ ». Il nous intéresse donc d'examiner dans quelle mesure nous sommes confrontés à des contre-langages, des contre-pouvoirs, qui tentent de rectifier les lignes de frontière, d'apporter, par exemple, de nouvelles puissances d'agir au menteur ou à la menteuse. Dans la littérature de femmes, lorsqu'une héroïne se met en scène et s'assume avec humour et souveraineté comme menteuse, il peut se produire ce phénomène de resignification. On peut constater, dans la deuxième moitié du $\mathrm{XX}^{\mathrm{e}}$ siècle, un certain engouement pour la variante féminine du picaro. Apparaissent aussi quelques imposteurEs (Hochstaplerinnen), quasiment absentes dans l'histoire littéraire en amont ${ }^{45}$.

Par conséquent, il faudrait établir si le recours au mensonge est une simple stratégie grâce à laquelle un personnage ou un-e auteur-e s'affranchit d'une morale dominante et agrandit l'espace vital de son ego (pour reprendre les termes de Vladimir Jankélévitch), ou si le recours au mensonge participe à une resignification: le menteur / la menteuse qui s'affiche menteur / menteuse, le fait-il/elle dans le but de revaloriser le mensonge?

\section{De la ruse d'Hérodiade à la danse de Salomé}

Le lien discursif entre mensonge et genre s'inscrit donc dans une historicité la femme vertueuse et transparente de Lessing, la grande hystérico-menteuse du $\mathrm{XIX}^{\mathrm{e}}$ siècle, la femme fatale de la Fin-de-siècle dans son usage du mensonge et de

${ }^{41}$ Judith Butler, Le pouvoir des mots. Politique du performatif, Paris, Éditions Amsterdam, 2004 [1 $1^{\text {re }}$ éd. Routledge, 1997].

${ }^{42}$ Aujourd'hui, queer ne s'oppose pas simplement à hétérosexuel, le terme vise même à perturber la dichotomie entre hétérosexualité et homosexualité. L'identité queer n'a pas de limites fermes, s'inscrit dans la fluidité, défie l'identique; c'est l'identité genrée postmoderne. Cf. Ibid., p. 39-40.

43 Voir par exemple la revue féministe Sorcières (1976-1981), dirigée par Xavière Gauthier.

44 ButLer, Le pouvoir des mots, p. 40.

${ }^{45}$ Cf. Dorothee Kiмmich, " "Mundus vult decipi". Warum man sich den Hochstapler als einen glücklichen Menschen vorstellen muss », Cahiers d'Études Germaniques, nº 67, p. 223-236. 
la ruse. Pour finir, la figure d'Hérodiade/ Salomé en littérature et arts permettra d'illustrer comment une construction du genre se modifie en lien avec l'interprétation de la ruse féminine dans un contexte franco-allemand, voire européen.

Alors que dans les textes bibliques, Salomé n'est même pas nommée ni sa danse décrite, elle devient un des mythes les plus populaires au tournant du siècle et sa danse l'une des représentations majeures de la femme fatale, tandis que la figure de la mère passe au second plan. La ruse d'Hérodiade, motivée initialement par le pouvoir, semblerait s'être déplacée, dans l'histoire complexe de sa réception, vers le corps de la danseuse incarnant la ruse autour de $1900^{46}$. Il faudrait donc ajouter aux deux éléments constitutifs du mythe de Salomé dans l'esthétique Fin-de-siècle - à savoir danse et violence ${ }^{47}$ - un troisième, la ruse faite corps, où l'on pourrait reconnaître non seulement des effets de mode - orientalisme et exotisme - mais aussi l'impact de l'hystérisation de la femme évoquée ci-dessus. Toutefois, étudier la "salomanie" de la période Fin-de-siècle, avec les accents différents de chaque interprétation, ainsi que les particularités nationales, dépasserait de loin le cadre de cette contribution. Notre analyse sur le déplacement du paradigme de la ruse d'Hérodiade se concentrera sur deux textes en amont dont l'impact sur le mythe a été considérable, à savoir l'Hérodiade de Heinrich Heine dans Atta Troll (1841) et celle de Gustave Flaubert dans Hérodias (1877), et montrera comment s'élaborent et se transforment les représentations du genre, ici en lien avec la ruse féminine.

Un petit rappel sur le personnage d'Hérodiade dans la Bible. Les passages chez Marc et Matthieu sont brefs, à peine quelques lignes chez ce dernier (Matth., 14, 1-12 et Marc, 6, 14-29). Salomé n'y joue qu'un rôle secondaire, elle exécute la volonté de la mère. Chez les deux Évangélistes, Hérodiade est la vraie coupable de l'emprisonnement de Jean-Baptiste et de sa décapitation. C'est elle qui n'aurait pas supporté les reproches proférés par celui-ci à l'égard de ses deux mariages incestueux. Hérodiade, humiliée, se serait sentie menacée par Jean-Baptiste, ce fauteur de troubles capable de discréditer sa position de pouvoir: « Hérodiade était acharnée contre lui. Elle aurait voulu le tuer mais ne le pouvait pas car Hérode, sachant que Jean était un homme juste et saint, le craignait et le ménageait. » (Marc, 6, 19-20) ${ }^{48}$. Lorsque Hérodiade, le jour du banquet, demande à Salomé de danser devant Hérode, elle utilise sciemment les charmes de sa fille comme instrument de sa ruse. Elle lui souffle le vœu fatal. C'est à elle que la fille rapporte le trophée: « Le garde [...] rapporta la tête sur un plat et la donna à la jeune fille, qui la donna à sa mère. » $(\text { Marc, } 6,28)^{49}$.

${ }^{46}$ Il serait également intéressant de mettre la danse de Salomé en perspective avec la naissance de la danse libre en Europe, avec sa revendication de naturel et d'authenticité. Loin de toute notion de ruse ou de tromperie associée au corps féminin, ces nouvelles formes de danse libre deviennent le nouveau paradigme de la vérité dans l'art.

${ }^{47} \mathrm{Cf}$. Sandra Walz, Tänzerin um das Haupt. Eine Untersuchung zum Mythos « Salome » und dessen Rezeption durch die europäische Literatur und Kunst des Fin de siècle, München, Martin Meidenbauer, 2008, p. XV. Voir deux autres ouvrages de référence sur la figure de Salomé en littérature et arts: Marion KocH, Salomes Schleier. Eine andere Kulturgeschichte des Tanzes, Hamburg, Europäische Verlagsanstalt, 1995, et Mireille Dottin-Orsini (dir.), Salomé, Paris, Autrement, 1996.

${ }^{48}$ La Bible (nouvelle traduction avec l'aide de nombreux écrivains dont François Bon, Emmanuel Carrère, Marie Depussé, Jean Echenoz, Marie Ndiaye, Valère Novarina...), Paris, Bayard, 2001, p. 2288.

49 Ibid. 
Les Pères de l'Église n'ont pas hésité à diaboliser Hérodiade, ainsi que sa fille, bien que celle-ci ait été encore longtemps représentée comme naïve et enfantine plutôt que perverse. Toutefois, Salomé ne devient Salomé, personnage autonome et incarnant elle-même la ruse initiale de la mère, qu'autour de 1900. Trois interprétations du mythe durant le $\mathrm{XIX}^{\mathrm{e}}$ siècle ont été déterminantes pour ce changement de paradigme: celle de Heinrich Heine, celle de Gustave Flaubert et celle d'Oscar Wilde. Leurs textes sont devenus le nouveau référent du mythe. De façon très schématique, on pourrait dire que $\mathrm{H}$. Heine a introduit le motif de l'amourpassion dans le mythe d'Hérodiade, que G. Flaubert a stylisé la danse et orientalisé Salomé, et qu'O. Wilde a concentré tout le potentiel érotique et cruel sur elle.

Hérodiade, dans la fable Atta Troll de Heine, écrite en 1841, fait figure de revenante. Au milieu du récit, le narrateur se trouve dans la cabane de la sorcière Uraka. Pendant que la sorcière tisse l'intrigue pour attirer l'ours Atta Troll, qui s'était échappé, dans un piège, le narrateur assiste, à la fenêtre, à une nuit de Sabbath dans laquelle apparaît Hérodiade, qui le charmera. Heine utilise ici les légendes médiévales qu'il a découvertes dans les œuvres des frères Grimm et dans lesquelles Hérodiade est devenue une sorcière hantant le monde des vivants. Mais, à la différence de ces légendes, il ne présente plus Hérodiade comme une femme laide, méchante et vengeresse ${ }^{50}$, équivalent du diable. Elle devient ambivalente et fascine par son mystère, par sa dangerosité ${ }^{51}$. Elle fascine notamment par la force de sa passion, car la ruse d'Hérodiade est désormais motivée par l'amour qu'elle aurait éprouvé à l'égard de Jean-Baptiste ${ }^{52}$.

Le motif du pouvoir politique est ainsi écarté. Au XIX ${ }^{\mathrm{e}}$ siècle, la femme ne peut avoir recours à une telle ruse que dans le cadre de l'amour. Bien que ce soit l'amour-folie que Heine cherche à valoriser, c'est toutefois une façon de situer la ruse d'Hérodiade à l'intérieur des rapports sociaux de sexes. Mais peut-on encore parler de ruse? Sur l'histoire qui a précédé la décapitation, nous apprenons très peu, et ce peu à quoi se réduit la ruse d'Hérodiade est davantage présenté comme un caprice féminin, d'autant plus arbitraire et inquiétant peut-être, mais vite regretté par la meurtrière elle-même ${ }^{53}$. Bien qu'on entende l'ironie chez Heine, il aura malgré tout préparé la réception ultérieure de la fille d'Hérodiade comme figure associant érotisme et violence. D'autres caractéristiques de son Hérodiade y contribuent: ses attributs physiques et vestimentaires orientalistes, le nom de Shéhérazade - autre figure de la ruse féminine, toutefois au service de la vie. Dans ses folles chevauchées nocturnes, Hérodiade emporte toujours le plateau d'argent avec la tête de Jean-Baptiste qu'elle embrasse avec passion, qu'elle jette même en l'air, comme un ballon, rieuse et ivre

${ }^{50}$ WaLz, Tänzerin um das Haupt, p. 100.

51 «Obs ein Teufel oder Engel,/ Weiß ich nicht. Genau bei Weibern/ Weiß man niemals, wo der Engel/ Aufhört und der Teufel anfängt. » Heinrich HeIne, Atta Troll. Ein Sommernachtstraum, in Sämtliche Schriften, vol. IV, München, Carl Hanser, 1971, p. 491-570, ici, p. 542 (Caput XIX).

52 « Denn sie liebte einst Johannem -/ In der Bibel steht es nicht,/ Doch im Volke lebt die Sage/ Von Hérodias' blutger Liebe -// Anders wär ja unerklärlich / Das Gelüste jener Dame -/ Wird ein Weib das Haupt begehren/ Eines Manns, den sie nicht liebt? » Ibid., p. 543 (Caput XIX).

53 «War vielleicht ein bißchen böse/ Auf den Liebsten, ließ ihn köpfen;/ Aber als sie auf der Schüssel/ Das geliebte Haupt erblickte,// weinte sie und ward verrückt,/ Und sie starb in Liebeswahnsinn. » Ibid. 
de jouissance, dans une scène assez grotesque. Puis elle lance un regard séducteur au narrateur qui s'enflamme pour elle et lui déclare son amour:

Doch mit toller Weiberlaune/ Schleudert sie das Haupt zuweilen/ Durch die Lüfte, kindisch lachend,/ Und sie fängt es sehr behende/ Wieder auf, wie einen Spielball. ${ }^{54}$ Liebe mich und sei mein Liebchen,/ Schönes Weib, Hérodias!// Liebe mich und sei mein Liebchen!/ Schleudre fort den blutgen Dummkopf/ Samt der Schüssel, und genieße/ Schmackhaft bessere Gerichte.// [...] Mich kümmerts wenig,/ Daß du tot und gar verdammt bist -55

Heine s'inscrit ainsi, non sans ironie à nouveau, dans la veine du romantisme noir qui érige la femme fatale en muse. Ni Salomé ni sa danse n’apparaissent. Mais, en revalorisant la figure d'Hérodiade comme déesse fascinante de par sa violence, il s'attaque surtout à l'idéologie chrétienne qui la diabolisa. Si quelqu'un a eu tort dans cette histoire, c'est bien Jean-Baptiste (ce « blutge[r] Dummkopf » = ce « benêt ensanglanté »), l'ascète qui a défendu une morale douteuse et qui a condamné la sensualité de la femme. C'est l'Éros ambivalent qui, chez Heine, remplace la ruse traîtresse. Ainsi, le terrain est préparé pour les représentations décadentes de la femme fatale autour de 1900, l'intrigue initiale d'Hérodiade perdant son importance ${ }^{56}$.

Heine semble avoir eu recours à ce mythe pour prendre le contre-pied de l'idéologie chrétienne, mais aussi pour s'identifier, en tant que juif exilé - il a écrit Atta Troll dans son exil à Paris - au sort de cette juive diffamée, qu'il représente certes errante, mais triomphant de toutes les morales médiocres qui tentent de briser son élan de vie et d'amour. C'est du moins ce que suggère $\mathrm{S}$. Walz, établissant une analogie entre les expériences du juif et de la femme:

Mit dem Bild der liebestollen Jüdin Hérodias, der sich der Mann nicht entziehen kann, forderte Heine die Arroganz der Zeitgenossen heraus. Heine versuchte eine Parallele zu schaffen zwischen der Intoleranz, die er als Jude erfahren hatte und der, welche die Frau in der patriarchalischen Gesellschaft des 19. Jahrhunderts erfahren musste. In der Hérodias-Figur scheinen sich die Erfahrungsbereiche des entwurzelten Künstlers mit denen der verfemten Sinnlichkeit zu vereinen. ${ }^{57}$

Pour nous, c'est un moment charnière où se modifie une représentation du genre en lien avec la ruse, et où l'on peut reconnaître un ressort important pour l'imaginaire de la Fin-de-Siècle ${ }^{58}$.

54 Atta Troll, p. 543-544 (Caput XIX).

55 Ibid., p. 547 (Caput XX).

${ }^{56}$ Il est toutefois intéressant de constater que l'apparition d'Hérodiade est précédée par d'autres figures féminines signifiant duplicité ou dangerosité de façon beaucoup plus stéréotypée: notamment la sorcière Uraka et son intrigue, tout comme Juliette dont le regard féminin séducteur est évoqué par le recours à la métaphore-cliché du piège: «Ihre Blicke sind ein süßes/ Strahlennetz, in dessen Maschen/ Unser Herz, gleich einem Fischlein,/ Sich verfängt und zärtlich zappelt. » Atta Troll, p. 499 (Caput I).

57 Walz, Tänzerin um das Haupt, p. 109.

58 « Durch jene, der Folklore-Tradition entliehene Thematisierung der Liebe zwischen Hérodias und Johannes wurde Heine für die Fin-de-siècle-Rezeption zum zentralen Impulsgeber, vor allem weil diese sinnliche Verrücktheit die Poesie widerspiegelte. Die Liebe, die Heine durch seine Hérodias offenbarte, war keine romantische Liebe mehr, sondern eher eine Parodie dessen, was unter dem luftigen Begriff "Liebe" verstanden wurde. Mit Hérodias entblätterte und entstaubte er den Liebebegriff und führte ihn zurück zu den Ursprüngen sinnlichen Begehrens und auch zu körperlicher Gewalt. ». Ibid., p. 109. 
Quant à la version de G. Flaubert, Hérodias, elle reste très près des sources bibliques puisque Flaubert y fait le portrait de la mère ambitieuse et de sa superbia offensée. La ruse d'Hérodias est au centre de la trame narrative. Flaubert rajoute même de la fiction romanesque pour renforcer ce portrait: «Depuis son enfance, elle nourrissait le rêve d'un grand empire. C'était pour y atteindre que, délaissant son premier époux, elle s'était jointe à celui-là, qui l'avait dupée, pensait-elle ${ }^{59} »$. Hérodias se sent dupée, puisque Hérode Antipas n'est pas à sa hauteur. Elle se moque de lui, de sa peur et méprise ses origines plébéiennes. L'implacable désir de pouvoir d'Hérodias est attesté par son sang-froid lorsqu'elle se félicite elle-même du succès de ses manigances pour écarter Agrippa, son propre frère ${ }^{60}$. Son «air d'impératrice» effraie le proconsul Vitellius qui la trouve «dangereuse ${ }^{61} »$. Parallèlement, Flaubert insiste sur les sentiments d'humiliation qui la torturent. Elle est « glacée par [1]es injures » proférées par Iaokanann en public - Flaubert garde le nom juif de Jean-Baptiste -. Ces critiques l'offensent profondément ${ }^{62}$, portent atteinte à sa gloire, menacent sa position de reine, puisque son mari si faible pourrait céder à l'opinion et la répudier ${ }^{63}$.

Enfin, par une subtile composition d'indices, Flaubert prépare la venue de Salomé au banquet en les identifiant comme éléments constitutifs de la ruse d'Hérodias, ourdie en réalité depuis bien longtemps. Hérodias aurait même fait éduquer sa fille à la danse pour un jour s'en servir comme arme ${ }^{64}$. Lorsque Hérode Antipas, dans le présent du récit, aperçoit une jeune fille au loin et lui demande qui elle est, Hérodias « répondit n'en rien savoir, et s'en alla soudainement apaisée ${ }^{65}$ ». Cette jeune fille apparaît une seconde fois de façon furtive, et de nouveau Hérodias ne répond pas à Hérode qui lui demande si c'est l'une de ses esclaves à elle. Puis elle apparaît une troisième fois, en entrant dans la salle du banquet, et alors Salomé se met à danser. Tout est calculé, y compris le moment où Salomé apparaît devant Hérode et les convives, la composition de la danse, l'effet érotique. Comme l'a observé Gabriele Brandstetter, la description de la danse chez Flaubert suit une véritable rhétorique corporelle de l'érotisme que Flaubert aurait composée à partir de son propre vécu en Égypte et à partir de représentations iconographiques médiévales ${ }^{66}$. Salomé commence par la danse du ventre, puis ses mouvements deviennent plus exaltés, frénétiques, « comme la rhombe des sorcières. [...] de ses vêtements jaillissaient d'invisibles étincelles qui enflammaient les hommes ${ }^{67} »$. Le public hurle, hystérisé. Puis, lors du dernier mouvement, Salomé, le corps renversé, les talons en l'air, parcourt l'estrade «comme un grand scarabée ». Elle s'arrête et fixe Hérode du regard. C'est à ce moment-là, capital, que se manifeste le dernier acte de la ruse d'Hérodias :

59 Gustave Flaubert, « Hérodias », in Trois contes, Paris, Folio, 1973, p. 108.

${ }^{60} «[\ldots]$ la joie d'un triomphe éclairait sa figure », ibid., p. 105.

${ }^{61}$ Ibid., p. 113.

62 « Iaokanann l'empêchait de vivre », ibid., p. 107.

${ }^{63}$ Ibid., p. 108.

${ }^{64}$ Ibid., p. 134.

65 Ibid., p. 109.

${ }^{66}$ Gabriele BrandstetTer, Tanz-Lektüren. Körperbilder und Raumfiguren der Avantgarde, Freiburg,

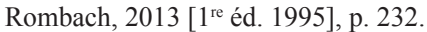

${ }^{67}$ Flaubert, Hérodias, p. 135. 
Un claquement de doigts se fit dans la tribune. Elle [Salomé] y monta, reparut; et, en zézayant un peu, prononça ces mots, d'un air enfantin: - Je veux que tu me donnes dans un plat, la tête... » Elle avait oublié le nom, mais reprit en souriant: "La tête de Iaokanann ${ }^{68}$ ! »

Flaubert fut un des premiers écrivains à avoir décrit la danse de Salomé. Il y mêla tout l'orientalisme de son époque. La réputation sulfureuse de la danseuse-prostituée sera par la suite un ingrédient majeur du mythe de Salomé. En 1891, c'est O. Wilde qui réinvestira avec sa Salomé cette aura lascive, érotique et orientaliste de Flaubert, mais avec un changement radical: désormais il n'est plus question de la mère, c'est Salomé elle-même qui se venge, parce que c'est elle qui aime Jean-Baptiste, c'est elle qui a été offensée dans son désir d'aimer. Sans doute, la figure de la mère ou de la sorcière Hérodiade ne convenait plus à cette génération de symbolistes et il fallait une figure plus juvénile pour incarner l'ambivalence d'une féminité mystérieuse et cruelle transfigurée en muse des poètes. L'implacable et terrifiante ruse d'Hérodiade, encore centrale chez Flaubert, sera passée entièrement dans le corps de la danseuse.

En guise de conclusion, nous voudrions évoquer certaines coïncidences dans l'histoire des gender studies et celle du mensonge. De nombreuses théoriciennes se sont prononcées pour une éthique relationnelle - Hélène Cixous l'appelle l'éthique de l'altérité, Luce Irigaray l'éthique de la différence sexuelle, Judith Butler l'éthique de la relation. La théorie d'une Cixous ou d'une Irigaray consiste à comprendre la différence sexuelle comme un différentiel, une dynamique où il est important d'accepter les failles, le secret, la part inéchangeable de l'échange, le fait qu'il y ait une altérité non appropriable. La vérité n'en est pas une, pourrait-on dire pour paraphraser Irigaray (Ce sexe qui n'en est pas un). J. Butler, quant à elle, critique dans le kantisme le refus de la part d'erreur et du mensonge dans l'humanité et oppose au sujet kantien, marqué par l'autonomie, la maîtrise et la souveraineté, un sujet éthique conscient de ses limites, interdépendant, toujours orienté vers et traversé par l'autre, et ayant droit à l'erreur ${ }^{69}$. Il nous semble que l'interrogation massive sur le genre telle qu'elle se pratique depuis maintenant un demi-siècle a, tout comme la réflexion psychanalytique, fortement impacté cette tendance à reconsidérer la perspective éthique sur le mensonge, mettant en avant la tache aveugle de nos relations, source d'erreurs mais aussi d'humanité.

${ }^{68}$ Ibid., p. 136.

69 Voir le commentaire de Doren Wohlleben sur l'éthique de la relation chez Judith ButLer, in Schwindel der Wahrheit, p. 230-231. 
